\title{
QUALIDADE PÓS-COLHEITA DE FRUTOS DE DIFERENTES CLONES DE MANGABEIRA (Hancornia speciosa GOMES) ${ }^{1}$
}

\author{
Postharvest quality of fruits from different mangabeira clones \\ (Hancornia speciosa GOMES)
}

\author{
Florisvaldo Gama de Souza², Raimundo Wilane de Figueiredo ${ }^{3}$, Ricardo Elesbão Alves ${ }^{4}$, \\ Geraldo Arraes Maia ${ }^{5}$ Ivaldo Antônio de Araújo ${ }^{6}$
}

\begin{abstract}
RESUMO
Com o objetivo de avaliar a qualidade pós-colheita de frutos de diferentes clones de mangabeira (Hancornia speciosa Gomes) para consumo como fruto fresco e/ou industrializado, foram avaliados 10 clones: Ipojuca 3, 4 e 5 (IPJ 3, 4 e 5); Nizia Floresta 1, 6 e 8 (NIF 1, 6 e 8); Parnamirim 11 (PAR 11); Rio Tinto 7 (RIT 7); Extremoz 1 (EXT 1) e Touros 48 (TOU 48), oriundos de um Jardim Clonal instalado em 1996 do Banco Ativo de Germoplasma da Estação Experimental de Mangabeira, pertencente a EMEPA Empresa Estadual de Pesquisa Agropecuária da Paraíba, situada em João Pessoa, PB. As mangabas foram colhidas manualmente em estádio de maturidade fisiológica, acondicionadas em caixas plásticas de transporte forradas no fundo com espuma de poliestireno e transportadas ao Laboratório de Fisiologia e Tecnologia Pós-colheita da Embrapa Agroindústria Tropical, onde foram caracterizadas física, físico-química e quimicamente. As características de qualidade analisadas foram: massa total do fruto; comprimento, diâmetro, firmeza, número de sementes; rendimento de polpa + casca; percentagem de sementes; pH; acidez total titulável (ATT); sólidos solúveis totais (SST); relação SST/ATT; vitamina C total; açúcares solúveis totais; açúcares redutores, fenólicos poliméricos, oligoméricos e dímeros. A análise de variância revelou diferenças estatísticas para todas as características pelo Teste F. De um modo geral os frutos de mangabeira dos clones avaliados apresentaram características superiores às observadas na literatura, especialmente quanto aos padrões estabelecidos pela I.N. $n^{\circ} 1$ de 07/01/2000 do MAPA. Dentre os materiais genéticos avaliados pode-se destacar os clones RIT 8 e TOU 48 como os mais promissores para o mercado de frutas in natura, e os clones IPJ 3 e NIF 6 para o processamento agroindustrial.
\end{abstract}

Termos para indexação: Pós-colheita, vitamina C, fenólicos, mangaba, Hancornia speciosa.

\section{ABSTRACT}

This work aimed to evaluate the postharvest quality of fruits from different mangabeira clones (Hancornia speciosa Gomes) for industrialization and/or in natura consumption. Fruits were analyzed from the following mangaba tree clones: Ipojuca 3, 4 and 5 (IPJ 3, 4 and 5); Nizia Floresta 1, 6 and 8 (NIF 1, 6 and 8); Parnamirim 11 (PAR 11); Rio Tinto (RIT 7); Extremoz 1 (EXT 1) and Touros 48 (TOU 48), grown as part of the germoplasma bank at Mangabeira Experimental Station of EMEPA - Empresa Estadual de Pesquisa Agropecuária da Paraíba, located at João Pessoa-PB, Brazil. Mangabas, were manually packed into polystyrene lined plastic boxes and transported to the Postharvest Physiology and Technology Laboratory at Embrapa Agroindústria Tropical, at Fortaleza-CE. At Embrapa, fruits were analysed for chemical and physical-chemical quality parameters. Quality parameters analyzed were weight, length and diameter, texture, number of seeds, yield pulp and skin, $\mathrm{pH}$, total titratable acidity (TTA), total soluble solids (TSS), TSS/TTA ratio, total vitamin C, total soluble sugars, reducing sugars, phenols as dimers, oligomers and polymers. Statistical analysis of variance revealed significant differences for all characteristics through Test F. Fruits from all the clones showed superior quality when compared to standards designed by MAPA on I.N. $n^{\circ} 1$ from 07/01/2000. Greatest industrial yields were observed for clones IPJ 3 and NIF 6. Clones RIT 7 and TOU 48 showed greater postharvest life suggesting potential for in natura consumption.

Index terms: Postharvest, vitamin C, phenolic compounds, mangaba, Hancornia speciosa.

\section{(Recebido em 23 de agosto de 2006 e aprovado em 31 de maio de 2007)}

\section{INTRODUÇÃO}

O mercado para mangaba encontra-se principalmente nas regiões Norte e Nordeste do Brasil. Em
Sergipe, é uma das frutas mais abundantes e procuradas nas feiras livres, atingindo preço superior ao da uva e de outras frutas nobres. Atualmente já ocorre a comercialização

\footnotetext{
'Parte da dissertação de mestrado do primeiro autor, apresentada ao curso de pós-graduação em Tecnologia de Alimentos

'Engenheiro de Alimentos, Mestre - Departamento de Tecnologia de Alimentos/DTA - Centro de Ciências Agrárias/CCA - Universidade Federal do Ceará/UFC Cx. P. 12168 - 60356-000 - florisvaldo.gs@gmail.com

${ }^{3}$ Engenheiro Agrônomo, Doutor, Professor - Departamento de Tecnologia de Alimentos/DTA - Centro de Ciências Agrárias/CCA - Universidade Federal do Ceará/UFC - Cx.P. 12168 - 60356-000 - figueira@ufc.br

${ }^{4}$ Engenheiro Agrônomo, Doutor, Pesquisador - Embrapa Agroindústria Tropical/CNPAT - Rua Doutora Sara Mesquita, 2270, Planalto do Pici - 60511-110 Fortaleza, CE - elesbao@cnpat.embrapa.br

${ }^{5}$ Engenheiro Agrônomo, Doutor, Pós-Doutorado - Departamento de Tecnologia de Alimentos/DTA - Centro de Ciências Agrárias/CCA - Universidade Federal do Ceará/UFC - Cx. P. 12168 - 60356-000 - gmaia@secrel.com.br

${ }^{6}$ Engenheiro Agrônomo, Mestre, Pesquisador - Empresa Estadual de Pesquisa Agropecuária da Paraíba/EMEPA - Rua Eurípedes Tavares, 210 , Tambiá 58013-590 - João Pessoa, PB - ivaldoaa@gmail.com
} 
em supermercados, em bandejas de isopor revestidas com filme de PVC com capacidade para 500g. Além do consumo in natura, o maior aproveitamento da mangaba se dá na forma de sucos, doces e sorvetes, sendo esse processamento feito por pequenas empresas e também por uma grande empresa produtora de sorvetes da região Nordeste (LEDERMAN et al., 2000).

As duas únicas coleções de germoplasma conhecidas pertencem a Empresa Pernambucana de Pesquisa Agropecuária - IPA e a Empresa Estadual de Pesquisa Agropecuária da Paraíba - EMEPA-PB, esta última é a maior com acessos oriundos de diferentes estados nordestinos (AGUIAR FILHO et al., 1998). A EMEPA dispõe de Banco Ativo de Germoplasma (BAG-Mangaba), iniciado em 1991 e que conta com aproximadamente 220 acessos. A partir do BAG em 1996, foram clonados 10 acessos com base na produção individual das plantas e formado um jardim clonal.

A identificação de materiais genéticos que, além de produtivos, apresentem qualidade superior para o aproveitamento industrial e/ou consumo in natura é de fundamental importância para formação de pomares (CHITARRA \& CHITARRA, 2005).

A necessidade de estudos sobre qualidade póscolheita de frutos de clones de mangabeira se faz óbvia, objetivando complementar as pesquisas realizadas sobre técnicas de propagação e cultivo, de modo que, uma vez implantados os pomares, os produtores estejam prontos a colocar os frutos no mercado em condições competitivas. Além disso, dispondo do conhecimento da qualidade e do potencial de utilização das frutas dos diferentes clones pode-se lançá-los de acordo com sua aptidão, ou seja, industrialização e/ou consumo in natura. Neste trabalho objetivou-se avaliar a qualidade pós-colheita de frutos de diferentes clones de mangabeira (Hancornia speciosa Gomes) para consumo in natura e/ou industrialização.

\section{MATERIAL E MÉTODOS}

As mangabas foram oriundas de plantas do Jardim Clonal da Estação Experimental de Mangabeira da Empresa Estadual de Pesquisa Agropecuária-EMEPA, João Pessoa, PB, constituído pelos seguintes clones: Extremoz (EXT 1), Ipojuca (IPJ 3, 4 e 5), Nizia Floresta (NIF 1, 6 e 8), Parnamirim (PAR 11), RioTinto (RIT 7) e Touros (TOU 48).

Os frutos foram colhidos manualmente em estádio de maturidade fisiológica, acondicionados em caixas revestidas com espuma no fundo e transportadas ao Laboratório de Fisiologia e Tecnologia Pós-colheita da Embrapa Agroindústria Tropical em Fortaleza-CE, onde foram caracterizadas fisicamente quanto à massa total $\mathrm{e}$ das sementes, rendimento de polpa + casca, número de sementes, comprimento, diâmetro e firmeza. A porção comestível, homogeneizada em centrífuga doméstica, foi avaliada quanto às seguintes características físico-químicas e químicas: Sólidos Solúveis Totais - SST ( ${ }^{\circ}$ Brix) conforme (AOAC, 1992), Açúcares Solúveis Totais - AST (YEMN \& WILLIS, 1954), Açúcares Redutores - AR (MILLER, 1959), Acidez Total Titulável - ATT (INSTITUTO ADOLFO LUTZ, 1985), SST/ATT obtido pelo quociente das duas variáveis, pH (AOAC, 1992), Vitamina C Total (STROHECKER \& HENNING, 1967) e fenólicos (REICHER et al., 1981).

$\mathrm{O}$ experimento foi realizado em delineamento experimental inteiramente casualizado com 10 tratamentos (clones), 22 repetições em média para as características físicas e 3 repetições para as físico-químicas e químicas. Para a caracterização física, os frutos foram considerados individualmente, enquanto para as físico-químicas e químicas as parcelas experimentais foram compostas pela polpa obtida de pelo menos 10 frutas.

Após a análise de variância dos dados obtidos, quando constatada a significância pelo teste $\mathrm{F}$, os tratamentos foram comparados pelo teste de REGWQ (Ryan Einot Grabiel Welsch Multuple Range) para as características físicas e pelo teste de Tukey para as características físico-químicas e químicas, ambos ao nível de $5 \%$ de probabilidade. As análises foram realizadas com auxílio dos programas computacionais SAS versão 8.0 e SISVAR versão 3.01, respectivamente.

\section{RESULTADOS E DISCUSSÃO}

Foram constatadas diferenças estatísticas para todas as características físicas, físico-químicas e químicas avaliadas (Tabelas 1 e 2).

As mangabas avaliadas apresentaram em média 25,74 g de massa total, sendo que o clone NIF 1 destacase em relação aos demais $(\mathrm{P}<0,05)$ com valor médio de 42,07 g. Estes resultados estão de acordo com os relatados na literatura para peso de frutos de mangabeiras nativas dos tabuleiros costeiros do Nordeste que varia 3 a $59 \mathrm{~g}$ (AGUIAR FILHO \& BOSCO, 1995; ALVES et al., 1989; GALDINO et al., 1996).

O rendimento médio da porção comestível (casca + polpa) das mangabas avaliadas foi relativamente alto, ou seja, aproximadamente $85 \%$. O clone EXT 1 apresentou o melhor rendimento - 88,88\%. Lemos (1988), trabalhando com frutos imaturos da mangabeira encontrou uma porcentagem média de $19 \%$ de casca, $71 \%$ de polpa e $10 \%$ de semente. Mosca (1996), trabalhando com frutos da 
mangabeira no Mato Grosso encontrou um valor de 80,63\% de polpa, $6,08 \%$ de casca e $13,29 \%$ de semente.

O comprimento oscilou entre 35,00 e 45,62 mm com média de $38,27 \mathrm{~mm}$. Na Tabela 1, o clone NIF 1 foi superior estatisticamente a todos os clones analisados, com valor equivalente a 45,62 $\mathrm{mm}$. A média obtida neste experimento ultrapassa o maior valor encontrado por Carvalho et al. (2003) em frutos da Região do Conde, BA. Por sua vez, Galdino et al. (1998) constataram um comprimento de 37,72 mm em mangabas do Litoral Paraibano, muito próximo à média citada previamente. Aguiar Filho et al. (1998) avaliaram plantas de diferentes regiões mantidas na mesma Estação Experimental deste trabalho e detectaram o maior comprimento para o clone Nizia Floresta $(35,70 \mathrm{~mm})$.

Conforme verifica-se na Tabela 1, o tratamento NIF 1 revelou um diâmetro de 40,41 mm, não diferindo estatisticamente dos clones NIF 8 (37,68 mm), RIT 7 (37,81 $\mathrm{mm})$ e TOU 48 (36,26 mm). O diâmetro médio observado por Ferreira et al. (2003) em pomar nativo de 30,60 mm e em pomar cultivado, $32,40 \mathrm{~mm}$, são inferiores aos detectados neste estudo. Os diâmetros encontrados têm apresentado uma medida menor em relação aos comprimentos, embora em muitos casos sejam próximos, fazem da mangaba um fruto com características piriformes, variando de oblongo a arredondado (PARENTE \& MACHADO, 1986).

A firmeza $(\mathrm{N})$ das mangabas oriundas dos diferentes clones avaliados foi bastante variável $(0,39$ a 4,53 N). Levando-se em consideração o mercado de fruta fresca os clones RIT 7 e TOU 48 destacam-se, tendo em vista que apresentaram firmeza de 4,52 e 4,35N, respectivamente.
O grau de resistência à pressão que as frutas maduras e "de vez" suportam nas medidas de textura, pode permitir inferências aos fabricantes de contentores a serem utilizados na colheita, transporte e armazenamento, ajudando-os a definir a máxima capacidade destes recipientes, em função do melhor rendimento na colheita e da máxima pressão que as frutas suportam sem sofrer danos físicos (SANTOS et al., 1999), isto demonstra a necessidade de se desenvolver e/ou adaptar caixas para colheita e comercialização de mangabas.

Frutos do clone NIF 1 revelaram um maior número de sementes, seguidos pelos clones RIT 7 e NIF 8 (Tabela 1) que corresponde a um percentual de sementes de $13,54 \%, 16,63 \%$ e $15,74 \%$, respectivamente. Apesar do clone NIF 1 possuir maior número de sementes, seu percentual de sementes foi inferior ao dos clones RIT 7 e NIF 8.

Araújo et al. (2003b) estudaram as medidas físicas e a produção de frutos do mesmo local de origem das mangabeiras deste experimento e os referidos autores encontraram na procedência NIF uma variação de 4 a 10 sementes por fruto; na IPJ de 6 a 10; na PAR de 8 a 11; na RIT de 5 a 12 e na EXT de 7 a 11, apresentando de forma geral resultados inferiores aos deste estudo, porém, sem tanta disparidade: NIF de 11,76 a 26,50; IPJ de 12,96 a 14,53; PAR com 13,4; RIT com 22,71 e EXT com 13,5 sementes por fruto. Em outra de suas pesquisas, Araújo et al. (2003a) citaram para o clone EXT um total de 11,22 sementes/frutos, dado comprovado neste estudo, com 11,09 sementes por fruto.

TABELA 1 - Características físicas de mangabas oriundas do Jardim da Clonal da EMEPA (João Pessoa, PB).

\begin{tabular}{llllllll}
\hline Clones & $\begin{array}{c}\text { Massa do } \\
\text { fruto }(\mathbf{g})\end{array}$ & $\begin{array}{c}\text { Comprimento } \\
(\mathbf{m m})\end{array}$ & $\begin{array}{c}\text { Diâmetro } \\
(\mathbf{m m})\end{array}$ & $\begin{array}{c}\text { Polpa+Casca } \\
(\boldsymbol{\%})\end{array}$ & $\begin{array}{c}\text { Sementes } \\
(\boldsymbol{\%})\end{array}$ & $\begin{array}{c}\mathbf{N}^{\mathbf{0}} \text { de } \\
\text { Sementes }\end{array}$ & $\begin{array}{c}\text { Firmeza } \\
(\mathbf{N})\end{array}$ \\
\hline EXT 1 & $23,22 \mathrm{bc}$ & $39,02 \mathrm{bc}$ & $32,40 \mathrm{c}$ & $88,88 \mathrm{a}$ & $11,13 \mathrm{a}$ & $11,09 \mathrm{~b}$ & $0,88 \mathrm{~b}$ \\
IPJ 3 & $23,25 \mathrm{bc} *$ & $37,95 \mathrm{bc}$ & $32,66 \mathrm{c}$ & $86,82 \mathrm{ab}$ & $13,18 \mathrm{bc}$ & $13,52 \mathrm{~b}$ & $1,34 \mathrm{~b}$ \\
IPJ 4 & $24,81 \mathrm{bc}$ & $38,73 \mathrm{bc}$ & $34,91 \mathrm{bc}$ & $84,26 \mathrm{bc}$ & $15,74 \mathrm{ab}$ & $14,53 \mathrm{~b}$ & $1,21 \mathrm{~b}$ \\
IPJ 5 & $21,74 \mathrm{c}$ & $35,01 \mathrm{c}$ & $32,57 \mathrm{c}$ & $81,63 \mathrm{c}$ & $18,37 \mathrm{a}$ & $12,96 \mathrm{~b}$ & $1,82 \mathrm{~b}$ \\
NIF 1 & $42,07 \mathrm{a}$ & $45,62 \mathrm{a}$ & $40,41 \mathrm{a}$ & $86,46 \mathrm{ab}$ & $13,54 \mathrm{bc}$ & $26,50 \mathrm{a}$ & $0,39 \mathrm{~b}$ \\
NIF 6 & $23,77 \mathrm{bc}$ & $37,87 \mathrm{bc}$ & $33,48 \mathrm{bc}$ & $86,13 \mathrm{ab}$ & $13,87 \mathrm{bc}$ & $11,77 \mathrm{~b}$ & $1,34 \mathrm{~b}$ \\
NIF 8 & $31,87 \mathrm{~b}$ & $38,90 \mathrm{bc}$ & $37,68 \mathrm{ab}$ & $84,26 \mathrm{bc}$ & $15,74 \mathrm{ab}$ & $22,14 \mathrm{a}$ & $1,13 \mathrm{~b}$ \\
PAR 11 & $24,24 \mathrm{bc}$ & $38,72 \mathrm{bc}$ & $34,46 \mathrm{bc}$ & $84,90 \mathrm{bc}$ & $15,10 \mathrm{ab}$ & $13,40 \mathrm{~b}$ & $2,23 \mathrm{~b}$ \\
RIT 7 & $32,08 \mathrm{~b}$ & $40,30 \mathrm{~b}$ & $37,81 \mathrm{ab}$ & $83,37 \mathrm{bc}$ & $16,63 \mathrm{ab}$ & $22,71 \mathrm{a}$ & $4,52 \mathrm{a}$ \\
TOU 48 & $27,05 \mathrm{bc}$ & $37,40 \mathrm{bc}$ & $36,26 \mathrm{abc}$ & $83,39 \mathrm{bc}$ & $16,61 \mathrm{ab}$ & $13,50 \mathrm{~b}$ & $4,35 \mathrm{a}$ \\
CV $(\%)$ & 32,52 & 11,96 & 12,65 & 3,89 & 21,94 & 44,06 & 106,02 \\
X Geral & 25,74 & 38,27 & 34,66 & 84,94 & 15,06 & 14,80 & 1,93 \\
\hline
\end{tabular}

* Médias seguidas pela mesma letra na vertical não diferem estaticamente entre si pelo Teste de Tukey $(\mathrm{P}<0,05)$. 
TABELA 2 - Características físico-químicas e químicas de mangabas oriundas do Jardim da Clonal da EMEPA (João Pessoa, PB).

\begin{tabular}{|c|c|c|c|c|c|c|c|c|c|c|}
\hline Clones & $\begin{array}{c}\text { SST } \\
\left({ }^{\circ} \mathrm{BRIX}\right)\end{array}$ & $\begin{array}{l}\text { AST } \\
(\%)\end{array}$ & $\begin{array}{l}\text { AR } \\
(\%)\end{array}$ & $\begin{array}{l}\text { ATT } \\
(\%)\end{array}$ & pH & $\begin{array}{c}\text { SST/AT } \\
\text { T }\end{array}$ & $\begin{array}{l}\text { FP } \\
(\%)\end{array}$ & $\begin{array}{l}\text { FO } \\
(\%)\end{array}$ & $\begin{array}{l}\text { FD } \\
(\%)\end{array}$ & $\begin{array}{c}\text { Vitamina C } \\
(\mathrm{mg} / \mathbf{1 0 0 g})\end{array}$ \\
\hline EXT 1 & $16,43 c$ & $16,59 \mathrm{abc}$ & $10,17 \mathrm{a}$ & $1,84 b c$ & $2,77 \mathrm{c}$ & $8,96 \mathrm{cde}$ & $0,32 \mathrm{bc}$ & $0,33 \mathrm{de}$ & $0,32 b$ & $161,90 \mathrm{bc}$ \\
\hline IPJ 3 & $19,37 \mathrm{a}$ & $18,53 \mathrm{a}$ & $8,73 \mathrm{abc}$ & $1,76 \mathrm{cde}$ & $2,97 \mathrm{abc}$ & $11,04 b$ & $0,36 a b$ & $0,39 \mathrm{abc}$ & $0,36 \mathrm{ab}$ & $160,47 b c$ \\
\hline IPJ 4 & $16,00 \mathrm{c}$ & $14,74 b c$ & $6,66 c$ & $2,07 a$ & $2,86 \mathrm{bc}$ & $7,75 \mathrm{e}$ & $0,35 \mathrm{abc}$ & $0,37 \mathrm{bcd}$ & $0,38 \mathrm{ab}$ & $139,61 \mathrm{c}$ \\
\hline IPJ 5 & $16,20 \mathrm{c}$ & $15,81 \mathrm{abc}$ & $8,81 \mathrm{abc}$ & $1,79 \mathrm{bcd}$ & $3,03 \mathrm{ab}$ & $9,05 \mathrm{cde}$ & $0,37 \mathrm{ab}$ & $0,40 \mathrm{ab}$ & $0,42 \mathrm{a}$ & $164,77 \mathrm{abc}$ \\
\hline NIF 1 & $17,23 b c$ & $14,48 \mathrm{c}$ & $8,84 a b c$ & $1,75 \mathrm{cde}$ & $3,01 \mathrm{ab}$ & $9,85 \mathrm{bcd}$ & $0,31 \mathrm{c}$ & $0,31 \mathrm{a}$ & $0,32 b$ & $160,89 b c$ \\
\hline NIF 6 & 19,30ab & $17,62 \mathrm{ab}$ & $9,60 \mathrm{ab}$ & $1,52 \mathrm{f}$ & $3,15 \mathrm{a}$ & $12,69 a$ & $0,38 \mathrm{a}$ & $0,41 a b$ & $0,41 \mathrm{a}$ & $167,98 \mathrm{ab}$ \\
\hline NIF 8 & $17,20 \mathrm{c}$ & $16,35 \mathrm{abc}$ & $9,02 \mathrm{abc}$ & 1,64def & $3,04 \mathrm{ab}$ & $10,45 b c$ & $0,40 \mathrm{a}$ & $0,41 a b$ & $0,42 \mathrm{a}$ & $159,88 b c$ \\
\hline PAR 11 & $16,67 \mathrm{c}$ & $16,57 \mathrm{abc}$ & $7,22 \mathrm{bc}$ & $1,93 \mathrm{ab}$ & $2,84 b c$ & $8,61 \mathrm{de}$ & $0,35 \mathrm{abc}$ & 0,35 cde & $0,36 \mathrm{ab}$ & $139,83 \mathrm{c}$ \\
\hline RIT 7 & $16,87 \mathrm{c}$ & $14,56 \mathrm{c}$ & $9,24 \mathrm{abc}$ & $1,76 \mathrm{cde}$ & $3,05 \mathrm{ab}$ & $9,60 \mathrm{bcd}$ & $0,36 a b$ & $0,37 \mathrm{abcd}$ & $0,38 \mathrm{ab}$ & $159,31 b c$ \\
\hline TOU 48 & $17,00 \mathrm{c}$ & $15,52 b c$ & $11,05 \mathrm{a}$ & $1,61 \mathrm{ef}$ & $3,17 \mathrm{a}$ & $10,54 b c$ & $0,38 \mathrm{a}$ & $0,42 \mathrm{a}$ & $0,40 \mathrm{a}$ & $188,75 \mathrm{a}$ \\
\hline $\mathrm{CV}(\%)$ & 4,20 & 6,42 & 10,12 & 3,25 & 2,58 & 5,78 & 4,94 & 4,43 & 6,21 & 5,51 \\
\hline X Geral & 17,23 & 16,08 & 8,94 & 1,77 & 2,99 & 9,85 & 0,36 & 0,37 & 0,38 & 160,34 \\
\hline
\end{tabular}

* Médias seguidas pela mesma letra na vertical não diferem estaticamente entre si pelo Teste de Tukey $(\mathrm{P}<0,05)$.

A maior variação do número de sementes ocorreu em frutos provenientes de Belém, PA, caracterizados por Mattietto et al. (2003) com mínimo de 5 e máximo de 40 sementes por fruto, média de 16 sementes/fruto.

A média geral encontrada para $\mathrm{pH}$ (Tabela 2) ficou abaixo dos valores mencionados por Mattietto et al. (2003) em frutos de Belém, PA, que foi de 3,46 e também por Moura et al. (2002), que foi de 3,29 para mangabas do Rio Grande do Norte, mas ligeiramente superior ao relatado por Mosca (1996) em frutos de mangabeiras nativas da Baixada Cuiabana, MT.

Com relação à acidez total titulável, os resultados não se apresentaram de forma homogênea, partindo em escala crescente de $1,52 \%$ observada no clone NIF 6 para $2,07 \%$, clone IPJ 4. A média foi equivalente a 1,77 \% idêntica a reportada por Moura et al. (2002), mesmo advindas de regiões diferentes. De acordo com Brasil (2000), o valor mínimo de acidez total titulável é de $0,7 \%$ para polpas de mangabas, conferindo a todo material analisado uma acidez pelo menos duas vezes maior superior. A baixa concentração indicada pelo Ministério da Agricultura e do Abastecimento em relação a dos clones analisados, se deve, provavelmente, à ação de um processo de diluição da polpa padronizada.

Analisando-se a Tabela 2, verifica-se que o clone NIF 6 com teor de 19,30 ${ }^{\circ}$ Brix não difere estatisticamente do IPJ 3 (19,37 ${ }^{\circ}$ Brix) e do NIF 1 (17,23 ${ }^{\circ}$ Brix), este último com concentração de sólidos solúveis totais igual ao da média geral de $17,23^{\circ}$ Brix. Os demais, também não diferiram estatisticamente entre si, apresentando-se teor de sólidos solúveis totais próximos da média geral referida. Todos os clones estudados encontram-se com teor de SST superiores ao mínimo padronizado pelo Ministério da Agricultura e do Abastecimento (Instrução Normativa $\mathrm{n}^{\circ}$ 01 de 07/01/2000) que é de $8^{\circ} \mathrm{Brix}$, provavelmente, porque a polpa de mangaba padronizada por este órgão tenha passado por um processo de diluição.

A relação SST/ATT apresentou uma oscilação de 7,75 a 12,69, com média geral de 9,85. Comparada com a literatura, ultrapassou a reportada por Moura et al. (2002) de 9,51 e foi menor do que a de Parente et al. (1985) com valores de 17,86 para frutos pequenos e médios de vez e 26,53 para frutos grandes de vez do cerrado da região Geoeconômica do Distrito Federal. A média geral constatada por Macêdo et al. (2003) foi de 10,64 para frutos oriundos da Estação Experimental de Mangabeira, João Pessoa, PB. Para o mercado de fruto fresco e/ou processado, a relação SST/ATT elevada é desejável. Neste contexto, a relação desenvolvida pelo clone NIF 6 revelou um valor de 12,69, diferindo-se estatisticamente dos outros tratamentos.

O teor de açúcares redutores apresentou uma equivalência entre os clones, porém, o TOU 48 foi o que maior valor revelou, com uma média igual a $11,05 \%$, seguido do EXT 1 com $10,17 \%$, contudo não diferem estatisticamente do IPJ 3 e 5, NIF 1, 6 e 8 e RIT 7. A quantidade de açúcares redutores encontrados por Moura et al. (2002) para mangabas provenientes do Rio Grande do Norte $(7,72 \%)$, ainda que menor, é semelhante a desta pesquisa. Os clones que indicam uma maior porcentagem de glicose e frutose em relação aos açúcares solúveis totais, 
são os mais preferíveis para o consumo in natura e industrialização, porque conferem aos frutos um sabor doce mais acentuado (BERNIZ, 1984).

De um modo geral, apesar das diferenças estatísticas encontradas, os frutos de mangabeira dos clones avaliados apresentaram valores médios dentro das faixas relatadas na literatura, com exceção de algumas características ainda pouco estudadas em mangabas como vitamina $\mathrm{C}$ e fenólicos.

O clone TOU 48 apresentou maior concentração de Vitamina C total (188,75 mg/100g), não havendo diferença significativa dos clones NIF $6(167,98 \mathrm{mg} / 100 \mathrm{~g})$ e IPJ 5 $(164,77 \mathrm{mg} / 100 \mathrm{~g})$. Os outros clones não diferiram estatisticamente entre si. Este resultado sugere ser a mangaba um fruto com destacado teor em Vitamina C, sobrepondo o encontrado por Alves et al. (2000) de 139,6 $\mathrm{mg} / 100 \mathrm{~g}$.

O teor de compostos fenólicos, em média de 0,37\%, é comparado ao encontrado no pedúnculo do cajueiro anão precoce, um fruto bastante adstringente, se ingerido in natura. O clone NIF 1 mostrou menor valor em todas as análises de fenólicos, onde foi obtido $0,31 \%$ para os compostos fenólicos poliméricos e oligoméricos, e 0,32\% para os dímeros. Pela análise estatística, este clone é semelhante aos clones PAR 11 e EXT 1. Alves et al. (2000) encontraram valores de $0,29 \%, 0,31 \%$ e $0,33 \%$, respectivamente, para os compostos fenólicos poliméricos, oligoméricos e dímeros.

\section{CONCLUSÕES}

De um modo geral os frutos de mangabeira dos clones avaliados apresentaram características superiores às observadas na literatura, especialmente quanto aos padrões estabelecidos pela I.N. $\mathrm{n}^{\circ} 1$ de 07/01/2000 do MAPA.

Dentre os materiais genéticos avaliados pode-se destacar os clones RIT 8 e TOU 48 como os mais promissores para o mercado de frutas in natura, e os clones IPJ 3 e NIF 6 para o processamento agroindustrial.

\section{REFERÊNCIAS BIBLIOGRÁFICAS}

AGUIAR FILHO, S. P. de; BOSCO, J. A mangabeira e sua importância para o Tabuleiro Costeiro Paraibano. Informativo SBF, Itajaí, v. 14, n. 4, p. 10, dez. 1995.

AGUIAR FILHO, S. P. de; BOSCO, J.; ARAÚJO, I. A. A mangabeira (Hancornia speciosa): domesticação e técnicas de cultivo. João Pessoa: Emepa-PB, 1998. 26 p. (Documentos, 24).
ALVES, R. E.; FILGUEIRAS, H. A. C.; MOURA, C. F. H. Caracterização de frutas nativas da América latina. Jaboticabal: Funep, 2000. 66 p. (Série frutas nativas, 9).

ALVES, R. E.; LEMOS, R. P.; OLIVEIRA, E. F.; SILVA, H.; SILVA, E. Q.; MALAVOLTA, E. Concentração de nutrientes na planta e nos frutos de mangabeira (Hancornia speciosa Gomes) por ocasião da colheita. In: CONGRESSO BRASILEIRO DE FRUTICULTURA, 10., 1989, Fortaleza. Anais... Fortaleza: SBF, 1989. p. 352-355.

ARAÚJO, I. A. de; FRANCO, C. F. de O.; BARROS NETO, M.; FONTINELLI, I. S. C. Caracterização física de frutos de progênies de mangabeira cultivadas no Litoral Paraibano. In: SIMPÓSIO BRASILEIRO SOBRE A CULTURA DA MANGABA, 2003, Aracajú. Anais... Aracajú: Embrapa Tabuleiros Costeiros, 2003a. CD-ROM.

ARAÚJO, I. A. de; FRANCO, C. F. de O.; MARINHO, S. J. O.; FERREIRA, E. G. Avaliações físicas e de produção de frutos do Banco Ativo de Germoplasma de Mangaba da EMEPA/PB no litoral paraibano. In: SIMPÓSIO BRASILEIRO SOBRE A CULTURA DA MANGABA, 2003, Aracajú. Anais... Aracajú: Embrapa Tabuleiros Costeiros, 2003b. CD-ROM.

ASSOCIATION OF OFFICIAL ANALYTICAL CHEMISTRY. Official methods of analysis of the Association of Official Analytical Chemistry. 12. ed. Washington, DC, 1992. $1115 \mathrm{p}$.

BERNIZ, P. J. Avaliação industrial de variedades de manga (Mangifera indica L.) para elaboração de néctar. 1984. 57 f. Dissertação (Mestrado em Ciência e Tecnologia de Alimentos) - Universidade Federal de Viçosa, Viçosa, 1984.

BRASIL. Ministério da Agricultura e do Abastecimento. Instrução normativa ${ }^{\circ} 1$, de 7 de janeiro de 2000. Diário Oficial [da] República Federativa do Brasil, Poder Executivo, Brasília, n. 6, p. 57, 10 jan. 2000. Seção 1.

CARVALHO, M. O. de; FONSECA, A. A. O.; SANTOS JÚNIOR, A. B. dos; HANSEN, D. de S.; RIBEIRO, T. de A. D. Caracterização física, organolépticas, química e físico-química dos frutos de mangabeiras (Hancornia speciosa Gomes) da região do Conde-BA. Disponível em: $<$ http://dados $\backslash$ frutalmangabal890.htm>. Acesso em: 29 jul. 2003. 
CHITARRA, A. B.; CHITARRA, M. I. F. Pós-colheita de frutos e hortaliças: fisiologia e manuseio. Lavras: UFLA, 2005.785 p.

FERREIRA, E. G.; SANTOS, E. S. dos; ARAÚJO, I. A. de; FRANCO, C. F. de O. Avaliações biométricas de plantas e físico-químicas de frutos de mangabeira de pomares nativo e cultivado. In: SIMPÓSIO BRASILEIRO SOBRE A CULTURA DA MANGABA, 2003, Aracajú, Anais... Aracajú: Embrapa Tabuleiros Costeiros, 2003. CD-ROM.

GALDINO, J. K. A.; SILVA, H.; SILVA, A. Q. da. Crescimento do fruto de mangaba (Hancornia speciosa) no litoral paraibano. In: CONGRESSO BRASILEIRO DE FRUTICULTURA, 14., 1996, Curitiba. Resumos... Curitiba: SBF, 1996. p. 316.

INSTITUTO ADOLFO LUTZ. Normas analíticas, métodos químicos e físicos para análise de alimentos. 3. ed. São Paulo, 1985. v. 1, 533 p.

LEDERMAN, I. E.; SILVA JÚNIOR, J. F.; BEZERRA, J. E. F.; ESPÍNDOLA, A. C. M. Mangaba (Hancornia speciosa Gomes). Jaboticabal: Ed. São Paulo, 2000. 35 p. (Série frutas nativas, 2)

LEMOS, R. P. de. Caracterização fenológica e teores de nutrientes da mangabeira (Hancornia speciosa Gomes). 1988. 44 f. Monografia (Graduação em Agronomia) Universidade Federal da Paraíba, Areia, 1988.

MACÊDO, L. de S.; ARAÚJO, I. A. de; FRANCO, C. F. de O. Caracterização físico-química de frutos da mangabeira nativa e naturalizada da Mata Paraibana. In: SIMPÓSIO BRASILEIRO SOBRE A CULTURA DA MANGABA, 2003, Aracajú. Anais... Aracajú: Embrapa Tabuleiros Costeiros, 2003. CD-ROM.

MATTIETTO, R. de A.; SOARES, M. da S.; RIBEIRO, C. C. Caracterização física e físico-química do fruto de mangaba (Hancornia speciosa Gomes) provenientes de Belém-PA. In: SIMPÓSIO BRASILEIRO SOBRE A CULTURA DA MANGABA, 2003, Aracajú. Anais... Aracajú: Embrapa Tabuleiros Costeiros, 2003. CD-ROM.
MILLER, G. L. Use of dinitrosalicylic acid reagent for determination of reducing sugars. Analytical Chemistry, Washington, v. 31, p. 426-428, 1959.

MOSCA, J. L. Caracterização de frutos de mangabeira (hancornia speciosa Gomes) nativas da Baixada CuiabanaMT. In: CONGRESSO BRASILEIRO DE FRUTICULTURA, 14., 1996, Curitiba. Resumos... Londrina: IAPAR, 1996. p. 314.

MOURA, C. F. H.; ALVES, R. E.; FILGUEIRAS, H. A. C.; ARAÚJO, N. C. C.; ALMEIDA, A. S. Quality of fruits native to latin america for processing: mangaba (Hancornia speciosa Gomes). Acta Horticulturae, Leuven, v. 2, n. 575, p. $549-554,2002$.

PARENTE, T. V.; BORGO, L. A.; MACHADO, J. W. B. Características químicas de frutos de mangaba (Hancornia speciosa Gomes) do cerrado da região geoeconômica do Distrito Federal. Ciência e Cultura, São Paulo, v. 37, n. 1, p. $96-98,1985$.

PARENTE, T. V.; MACHADO, J. W. B. Germinação de sementes de mangaba (Hancornia pubescens Nees e Mart.) provenientes de frutos colhidos com diferentes graus de maturação. Revista Brasileira de Fruticultura, Cruz das Almas, v. 8, n. 1, p. 39-43, ago. 1986.

REICHER, F.; SIERAKOWSKI, M. R.; CORRÊA, J. B. C. Determinação espectrofotométrica de taninos pelo reativo, fosfotúngstico-fosfomomolíbdíco. Arquivos de Biologia e Tecnologia, Curitiba, v. 24, n. 4, p. 401-411, 1981.

SANTOS, A. R. L. dos; REINHARDT, D. H.; SILVEIRA, W. R.; OLIVEIRA, J. R. P.; CALDAS, R. C. Qualidade póscolheita de acerola para processamento, em função de estádios de maturação e condições de armazenamento. Revista Brasileira de Fruticultura, Jaboticabal, v. 21, n. 3, p. 365-371, dez. 1999.

STROHECKER, R.; HENNING, H. M. Analisis de vitaminas: métodos comprobados. Madrid: Paz Montalvo, 1967. 428 p.

YEMN, E. W.; WILLIS, A. J. The estimation of carbohydrate in plant extracts by anthrone. The Biochemical Journal, London, v. 57, p. 508-514, 1954. 